\title{
ERRATA
}

\section{VOLUME 5}

Page 58, line 7. In the second formula in the line, replace inferior $九$ by (๘).

Page 58, line 8. In the second formula in the line, replace inferior $\iota$ by ( $\iota)$, three times.

Page 58, line 9 from the bottom. Insert a parenthesis) immediately before the period at the end of the line.

Page 66, lines 16 and 17. These lines should read: "Now by substitution in $16^{\alpha^{\prime}}$,

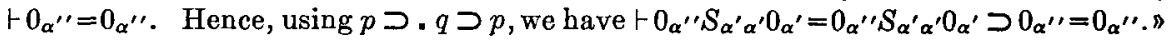

Page 66, line 22, at the end of the line. Replace inferior $\alpha$ by $\alpha^{\prime \prime}$.

Page 111, line 23. Replace $\sim(A \bar{B})$ by $\sim \diamond(A \bar{B})$.

Page 120, line 19. For "indirrizzi», read «indirizzi».

Page 154. In the signature of the review, for "ARHUR 》, read "ARTHUR 》.

Page 179, line 17. In the Index of reviews by subjects, the following additions should be made to the entry Deduction theorem: $3825,2857,28513,4422,3966,3849$.

\section{VOLUME 6}

Page 38, line 20. For « Herman 》, read « Hermann》.

Page 39, line 15. For «J. L. 》, read «L. J. ».

Page 48, line 7. For $\mathbf{R}^{\rho}$, read $\mathbf{Q}^{\rho}$.

Page 55, footnote 9 , line 2. Transpose $\lambda x(\mathfrak{M N})$ and $(\lambda x \mathfrak{M}) \mathfrak{R}$.

Page 56, Insert parentheses in the definientia of $S_{\lambda}$, etc., so that these definitions become:

$$
\begin{aligned}
S_{\lambda} & \equiv \lambda x \lambda y \lambda z(x z(y z)) . \\
K_{\lambda} & \equiv \lambda x \lambda y x . \\
B_{\lambda} & \equiv \lambda x \lambda y \lambda z(x(y z)) . \\
C_{\lambda} & \equiv \lambda x \lambda y \lambda z(x z y) . \\
W_{\lambda} & \equiv \lambda x \lambda y(x y y) .
\end{aligned}
$$

Page 68, line 6 from the bottom. For " LANGFOPD ", read "LANGFord".

Page 72, line 30. For "ERnest ", read "Ernst ".

Page 107, line 21. Transpose $k$ and $h$, so that last word of the title becomes "Hypothesenwahrscheinlichkeit" $"$

Page 109, line 16. For « Socrages ", read "Socrates".

Page 115, line 5. The clause following the comma should read: "the last symbol in each of the last three brackets should be $\alpha_{3}^{\prime \prime \prime}$ rather than $\alpha_{3}^{\prime \prime}$." 